\title{
VALIDAÇÃO DE MÉTODO ANALÍTICO PARA DETERMINAR A MIGRAÇÃO DE $\varepsilon$-CAPROLACTAMA DAS EMBALAGENS PARA ALIMENTOS GORDUROSOS
}

\author{
Marcus Vinicius Justo Bomfim e Shirley de Mello Pereira Abrantes* \\ Departamento de Química, Instituto Nacional de Controle de Qualidade em Saúde/Fundação Oswaldo Cruz, Av. Brasil, 4365, \\ 21040-900 Rio de Janeiro - RJ, Brasil \\ Helena Pereira da Silva Zamith \\ Departamento de Farmacologia e Toxicologia, Instituto Nacional de Controle de Qualidade em Saúde/ Fundação Oswaldo Cruz,
} Av. Brasil, 4365, 21040-900 Rio de Janeiro - RJ, Brasil

Recebido em 1/9/09; aceito em 18/1/10; publicado na web em 3/5/10

\begin{abstract}
VALIDATION OF ANALYTICAL METHOD TO DETERMINE $\varepsilon$-CAPROLACTAM MIGRATION FROM PACKAGINGS TO FATTY FOOD. $\varepsilon$-Caprolactam (CAP) is a monomer of nylon 6 used as food packaging for bologna sausage, turkey blanquettes, fowl breast, pâtés and ham luncheon meat. After polymerization a part of the monomer can remain in the packaging and migrate into the food. The aim of this work was develop and validate a single laboratory method to determine CAP in ethanol $95 \%$ that simulates fatty food characteristics. Thus, linear range was 2 to $32 \mathrm{mg} / \mathrm{L}$ of CAP, detection and quantification limits were 0.83 and $1.63 \mathrm{mg} / \mathrm{L}$, respectively. Repeatability showed Hor Rat values lower than 2 while recovery range was 97.5 to $106.5 \%$. The method was considered adequate for purpose.
\end{abstract}

Keywords: caprolactam; nylon 6; validation.

\section{INTRODUÇÃO}

A ع-caprolactama (CAP), azepan-2-ona (CAS 105-60-2), uma amida-cíclica, é um monômero precursor de polímeros denominados nylon 6. São utilizados na produção de tapetes, vestuário e materiais plásticos (equipamentos, sistemas e componentes automotivos, conectores, além de embalagens plásticas). Quando o nylon 6 tem sua aplicação no setor de embalagens, particularmente aquelas dirigidas ao segmento de alimentos gordurosos, existe uma preocupação relacionada à produção do polímero. Como a reação de polimerização não possui um rendimento de $100 \%$, resíduos do monômero CAP (Figura 1) podem permanecer adsorvidos à macromolécula de nylon 6 e migrar, por difusão, para o alimento.1,2 A literatura disponível sobre a migração de CAP em filmes de nylon 6 para alimentos e simulantes é restrita. Os valores de migração de CAP nas embalagens multicamadas contendo nylon 6 utilizadas para queijo e produtos de origem animal variaram de 6,4 a $10 \mathrm{mg} / \mathrm{kg}$ em simulante etanol $95 \% .3$ Diversos produtos contendo nylon 6 em sua constituição apresentaram níveis de migração da CAP entre 6,4 e $42,7 \mathrm{mg} / \mathrm{kg}$ para etanol 95\%.4 Além disso, são inúmeros os trabalhos científicos acerca da toxicidade da CAP. Os estudos de genotoxicidade in vitro e in vivo por via oral e intraperitoneal mostram, em sua grande maioria, resultados negativos bem como ausência de efeitos carcinogênicos em ratos e camundongos e sobre o desenvolvimento e reprodução em ratos e coelhos. Estudos epidemiológicos sugerem que a CAP pode causar hipotensão, taquicardia, palpitações, ressecamento nasal, efeitos geniturinários e sobre a reprodução como distúrbios nas funções menstrual e ovariana, e complicações no parto; além de problemas neurológicos e hematológicos.5 Diante da possibilidade de ocorrência de eventos toxicológicos originados de exposição oral ou intraperitoneal à CAP, a legislação brasileira estabelece limite de migração específica para a CAP de $15 \mathrm{mg} / \mathrm{kg}$ ou L de alimento ou simulante.6

Quaisquer laboratórios envolvidos no processo e dinâmica de controle e fiscalização necessitam desenvolver instrumentos ou meios para cumprir com suas atribuições. O principal deles diz respeito ao desenvolvimento de métodos analíticos para identificação e quantificação de substâncias. É imprescindível, contudo, que sejam comprovadas a eficiência, confiabilidade e adequabilidade dos procedimentos analíticos e de seus resultados para a tomada de qualquer tipo de decisão. Nesse contexto, é internacionalmente reconhecido que laboratórios devam tomar medidas apropriadas para assegurar que são capazes de produzir resultados com a qualidade requerida, como utilização de métodos de ensaio validados. ${ }^{7}$

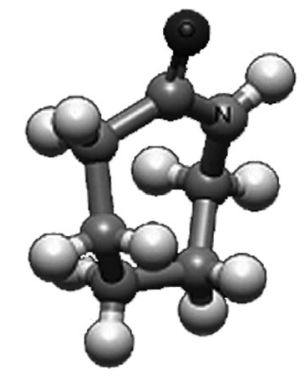

Figura 1. Estrutura tridimensional da molécula da ع-caprolactama ${ }^{8}$

A validação intralaboratorial é internacionalmente reconhecida como alternativa quando não há disponibilidade de procedimento interlaboratorial ou quando os procedimentos não são aplicáveis. ${ }^{7}$ A validação intralaboratorial diz respeito a estudos analíticos que envolvem um único laboratório, utilizando um mesmo método para analisar a mesma amostra, sob diferentes condições, em um intervalo de tempo justificado. ${ }^{9}$ Como inexistem estudos colaborativos para o método de ensaio aqui proposto, o estudo adotou procedimentos característicos de uma validação intralaboratorial.

Este estudo teve como objetivo desenvolver e validar um método analítico para detecção e quantificação de resíduos de CAP provenientes de embalagens de nylon 6 e transferidos aos alimentos, particularmente os gordurosos. 


\section{PARTE EXPERIMENTAL}

\section{Amostras e reagentes}

As amostras são embalagens multicamadas de nylon 6, mais precisamente três filmes de nylon, destinadas a acondicionar alimentos gordurosos. Os alimentos gordurosos incluídos no escopo do estudo foram a mortadela, o blanquet de peru, peito de aves, patês e apresuntado. Esses alimentos apresentam uma particularidade: o processo de cozimento é realizado dentro da embalagem sob temperaturas que variam de 70 a $100{ }^{\circ} \mathrm{C}$, num período entre 1 a $4 \mathrm{~h}$ ou até que o centro do alimento alcance a temperatura de $72{ }^{\circ} \mathrm{C}$.

O material utilizado para o procedimento de validação analítica foi fornecido por empresa reconhecida do setor de embalagens. A amostra, denominada AMV, era constituída de camadas de nylon 6/ adesivo/nylon 6/adesivo/nylon 6 (80\%) e nylon 6/6,6 (20\%).

A CAP (pureza $>99 \%$, Sigma-Aldrich) foi utilizada como padrão analítico e o etanol $99 \%$ (pureza $>99,9 \%$, grau para cromatografia líquida de alta eficiência) foi adquirido da Merck para ser utilizado como simulante após a diluição em água destilada.

\section{Método de ensaio}

O método de ensaio fundamenta-se na transferência de resíduos de CAP presentes em algumas embalagens de alimentos para um simulante que imita as características dos alimentos. Aqui, o ensaio de migração propõe o contato de $1 \mathrm{dm}^{2}(10 \times 10 \mathrm{~cm}) \mathrm{do}$ material com $100 \mathrm{~mL}$ do simulante selecionado, etanol 95\%, em placas de Petri de $15 \mathrm{~cm}$ de diâmetro. O ensaio foi conduzido por imersão total do material que permaneceu à temperatura de $60 \pm 0,5{ }^{\circ} \mathrm{C}$ durante $3 \mathrm{~h} 30 \mathrm{~min}$. As condições de realização do ensaio basearam-se na Diretiva Européia n ${ }^{\circ} 711$, de 18/10/1982, e no documento da Agência Nacional de Vigilância Sanitária, ainda em consulta pública, que substituirá a Resolução n ${ }^{\circ} 105$, de 19/5/1999. ${ }^{10,11}$ Esses estabelecem que para alimentos submetidos ou elaborados em temperaturas entre 70 e $100^{\circ} \mathrm{C}$, e por período de $1 \mathrm{a} 4 \mathrm{~h}$ (como no caso das embalagens em questão), as condições de realização do ensaio com simulante etanol $95 \%$ devam ser aquelas adotadas neste trabalho.

A solução resultante do ensaio de migração contendo os resíduos da CAP em etanol $95 \%$ foi transferida a um balão volumétrico e completado o seu volume a $100 \mathrm{~mL}$. Frascos de $2 \mathrm{~mL}$ foram preenchidos com tal solução para posterior injeção no equipamento.

\section{Equipamento}

Utilizou-se o cromatógrafo a gás Shimadzu GC-2010 acoplado a um detector por ionização em chama (DIC), com a coluna de sílica fundida recoberta internamente com fase estacionária constituída de $5 \%$ fenilmetilsilicone (HP-5 da J\&W Scientific) com as dimensões de $30 \mathrm{~m}$ de comprimento $\mathrm{x} 0,53 \mathrm{~mm}$ de d.i. $\mathrm{x}$ 2,65 $\mu \mathrm{m}$ de espessura de filme interno. As injeções foram executadas por autoinjetor automático Shimadzu modelo AOC-20i.

\section{Condições cromatográficas}

As condições de operação do equipamento foram determinadas e otimizadas utilizando-se a coluna HP-5. As temperaturas do injetor, coluna e detector foram, respectivamente, $210^{\circ} \mathrm{C} ; 100^{\circ} \mathrm{C}(1 \mathrm{~min})$ até $180{ }^{\circ} \mathrm{C}(3 \mathrm{~min})$, com acréscimo de $4{ }^{\circ} \mathrm{C} / \mathrm{min}$ e $250^{\circ} \mathrm{C}$. O gás de arraste hélio foi mantido à pressão de $60 \mathrm{kpa}$, com vazão na coluna de $10,14 \mathrm{~mL} / \mathrm{min}$. O tempo de análise foi de $24 \mathrm{~min}$, sendo injetado um volume de $2 \mu \mathrm{L}$ no cromatógrafo.

\section{Soluções estoque e de trabalho}

As soluções estoque de CAP foram preparadas em etanol 95\% na concentração de $1000 \mathrm{mg} / \mathrm{L}$. Posteriormente, por diluição, foram obtidas soluções de trabalho conforme a necessidade. As soluções foram armazenadas sob refrigeração entre 6 e $10^{\circ} \mathrm{C}$ por período de 1 ano.

\section{Validação intralaboratorial}

O procedimento para validação intralaboratorial foi basicamente descrito por Souza. ${ }^{12} \mathrm{~A}$ adequação ao propósito do método foi determinada a partir dos resultados de parâmetros como linearidade, efeitos de matriz, recuperação, repetitividade, precisão intermediária, limites de detecção e quantificação. Salienta-se que a precisão intermediária e os limites de detecção e quantificação foram determinados segundo INMETRO e ICH; e Frehse e Thier, respectivamente. ${ }^{13-15}$

Todo o tratamento estatístico necessário foi realizado com auxílio do programa Microsoft Office Excel 2000.

\section{Linearidade}

A linearidade foi realizada através de ensaios com soluções padrão. Preparou-se uma curva analítica, com níveis de concentração igualmente espaçados $2 ; 8 ; 14 ; 20 ; 26$ e $32 \mathrm{mg} / \mathrm{L}$, sendo três repetições independentes de cada nível.

A linearidade foi avaliada através da estimativa dos parâmetros pelo método dos mínimos quadrados ordinários (MMQO), inspeção visual dos dados e gráficos e tratamento de valores aberrantes. Os valores aberrantes foram tratados pelo teste de resíduos padronizados Jacknife, o qual foi aplicado consecutivamente até que novos valores aberrantes não fossem detectados ou até exclusão máxima de $22,2 \%$ do número de análises. ${ }^{16,17}$

O MMQO parte da premissa que os resíduos seguem a distribuição normal, têm variância constante ao longo do eixo x e são independentes. Tais premissas relacionadas à análise de regressão foram avaliadas quanto à normalidade (pelo teste de Ryan-Joiner); homoscedasticidade (pelo teste de Levene modificado por Brown e Forsythe) e independência dos resíduos da regressão (teste de Durbin \& Watson). ${ }^{18-21} \mathrm{O}$ teste $\mathrm{F}$ foi conduzido para verificar o ajuste ao modelo linear por meio da avaliação das significâncias da regressão e do desvio de linearidade avaliado contra o erro puro. ${ }^{22}$

\section{Efeito matriz}

A avaliação do efeito de matriz proposta por Souza sugere a confecção de duas curvas do analito: uma, denominada de solventes, originada a partir de soluções com padrão, e outra, denominada de matriz, originada de amostras. ${ }^{12}$

O método proposto sugere a utilização de amostra branca ou material de referência certificado (MRC). ${ }^{12}$ Porém, diante da dificuldade de obtenção de tais amostras, as soluções de CAP foram adicionadas à solução resultante do ensaio de migração da amostra $\mathrm{AMV}$. $\mathrm{O}$ valor da migração da CAP obtido para a amostra AMV foi determinado a partir da média de áreas dos picos cromatográficos originados de 20 ensaios de migração com a mesma.

A curva da matriz foi checada pelo método de adição. Alíquotas de solução de CAP foram adicionadas à solução resultante do ensaio de migração da amostra AMV em níveis de concentração equivalentes a 2; 8; 14;20;26 e $32 \mathrm{mg} / \mathrm{L}$. Para cada concentração foram realizadas três repetições independentes.

As áreas produzidas pelos ensaios de migração foram, então, subtraídas da média de áreas dos picos cromatográficos para amostra $\mathrm{AMV}$, objetivando encontrar somente a resposta relativa às soluções de adição. Tais valores foram utilizados em todo tratamento estatístico e na confecção da curva analítica da matriz. 
Os dados experimentais obtidos para as curvas do analito em solvente e matriz foram analisados pelo MMQO, sendo obrigatório o atendimento das premissas citadas na avaliação da linearidade. A seguir realizou-se, através do teste de hipóteses, a confirmação de que as variâncias dos resíduos da regressão eram homogêneas. Sendo homogêneas, procedeu-se à comparação entre as inclinações e interseções das curvas de solventes e de matriz através do teste $t$ com variâncias combinadas.

\section{Repetitividade e recuperação}

A repetitividade e a recuperação sob condições equivalentes (mesmo analista, mesmo equipamento, mesmo laboratório, mesmos reagentes e curto período de tempo) foram avaliadas por ensaios de migração com a amostra AMV. Tais amostras foram submetidas ao ensaio de migração e, posteriormente, adicionadas por alíquotas em níveis de concentração correspondentes a 2; 8; 14; 20;26 e 32 mg/L, com pelo menos cinco repetições genuínas de cada nível, sendo dois níveis por dia. As alíquotas foram tomadas como se a amostra AMV não apresentasse qualquer resíduo de CAP.

\section{Precisão intermediária}

Os experimentos foram conduzidos em três diferentes dias, permitindo avaliar a precisão intermediária através do $C V(\%)$. O coeficiente de variação foi obtido a partir do desvio padrão da precisão intermediária e da média das mensurações dos três dias, sendo 10 ensaios por dia.

Limites de detecção (LOD) e quantificação (LOQ)

Os limites foram estimados a partir da inclinação e interseção da curva analítica como sugerido por Frehse e Thier. ${ }^{15}$

\section{RESULTADOS E DISCUSSÃO}

\section{Linearidade e curva analítica}

A utilização do MMQO exige a confirmação das premissas do método. As premissas devem ser satisfeitas, caso contrário, o MMQO não pode ser realizado. Estas se relacionam aos resíduos da regressão e dizem respeito a testes de normalidade, independência e homogeneidade das variâncias dos resíduos, além da análise de variância da regressão e desvio da linearidade dos resíduos. A Tabela 1 apresenta os resultados dos referidos testes.

Tabela 1. Resultados dos testes de premissas para avaliação da linearidade e confecção da curva analítica $(\alpha=0,05)$

\begin{tabular}{lccc}
\hline Testes estatísticos & Estatística & Padrão de CAP & Valor crítico \\
\hline Normalidade & $R$ & 0,9755 & $>0,9351$ \\
Homoscedasticidade & $t_{L} ; p$ & 0,$274 ; p=0,79$ & $<2,18 ; p>0,05$ \\
Independência & $d$ & 1,52 & $>d_{L}=1,05 ;>d_{U}=1,35$ \\
Regressão & $p$ & $1,48 \times 10^{-18}$ & $<0,001$ \\
Desvio da linearidade & $p$ & $9,31 \times 10^{-1}$ & $>0,05$ \\
\hline
\end{tabular}

$R=$ coeficieente de correlação de Ryan - Joiner; $t_{L}=$ estatística $t$ de Levene; $d=$ estatística de Durbin - Watson; $p=$ significância

Satisfeitas as premissas do método, o MMQO foi utilizado para confecção e ajuste da curva analítica. Ao final, a linearidade do método foi comprovada na faixa de trabalho de 2 a $32 \mathrm{mg} / \mathrm{mL}$. A equação final de regressão foi $y=6651,9+17521 x$, sendo $y$ a área do pico da CAP e $x$ a concentração do analito (Figura 2).

\section{Efeito matriz}

A solução teste originada pelo ensaio de migração da amostra AMV foi adicionada nas concentrações de trabalho da curva

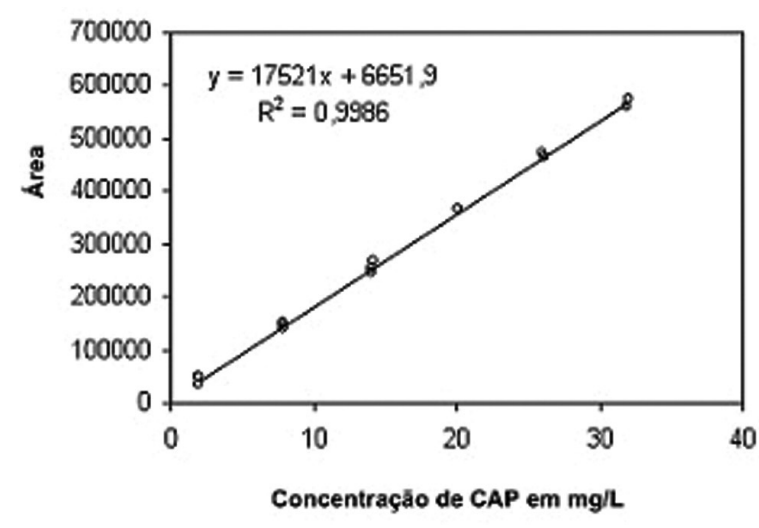

Figura 2. Curva analítica da $\varepsilon$-caprolactama (CAP) em etanol $95 \%$

analítica. As respostas obtidas foram subtraídas da área média da amostra AMV. A resposta média (área) e a migração estimada para amostra AMV foram de 142087,5 e 7,73 mg/L, respectivamente. Os resultados dos testes de premissas bem como a curva analítica da CAP em matriz podem ser visualizados na Tabela 2 e na Figura 3 , respectivamente.

Tabela 2. Testes de premissas para curva analítica em matriz $(\alpha=0,05)$

\begin{tabular}{lccc}
\hline Testes estatísticos & Estatística & Matriz & Valor crítico \\
\hline Normalidade & $R$ & 0,9880 & $>0,9383$ \\
Homoscedasticidade & $t_{L} ; p$ & 0,$302 ; p=0,77$ & $t_{L}<2,16 ; p>0,05$ \\
Independência & $d$ & 2,05 & $>d_{L}=1,08 ;>d_{L U}=1,36$ \\
Regressão & $p$ & $1,06 \times 10^{-16}$ & $<0,001$ \\
Desvio da linearidade & $p$ & 0,60 & $>0,05$ \\
\hline
\end{tabular}

$R=$ coeficieente de correlação de Ryan - Joiner; $t_{L}=$ estatística $t$ de Levene; $d=$ estatística de Durbin - Watson; $p=$ significância

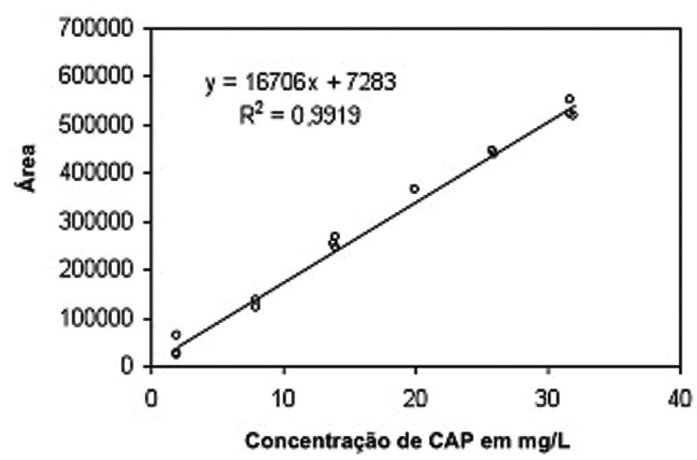

Figura 3. Curva analítica da $\varepsilon$-caprolactama (CAP) em matriz.

Satisfeitas as premissas e confirmada a linearidade do analito em matriz, as etapas subsequentes como o teste de homoscedasticidade das variâncias dos resíduos e o teste $t$ para variâncias combinadas

Tabela 3. Resultados dos testes para avaliação do efeito matriz $(\alpha=0,05)$

\begin{tabular}{lccc}
\hline Testes estatísticos & Estatística & Calculado & Valor crítico \\
$\begin{array}{l}\text { Homoscedasticidade das } \\
\text { variâncias dos resíduos }\end{array}$ & $F$ & 0,16 & $<2,06$ \\
$\begin{array}{l}\text { Teste t para variâncias } \\
\text { combinadas }\end{array}$ & $t_{b} ; t_{a}$ & $\begin{array}{l}t_{b}=1,75 \\
t_{a}=0,07\end{array}$ & $<2,06$ \\
\hline
\end{tabular}

$F=$ razão entre as variâncias dos resíduos de solvente e matriz; $t_{b}=$ estatística $t$ para comparação das inclinações das curvas de solvente e matriz; $t_{a}=$ estatística $t$ para comparação das intersecções das curvas de solvente e matriz 
Tabela 4. Avaliação da repetitividade e recuperação do método para cada nível de concentração

\begin{tabular}{lcccc}
\hline $\begin{array}{l}\text { Critérios de } \\
\text { desempenho }\end{array}$ & Estatística & $\begin{array}{c}\text { Concentração } \\
(\mathrm{mg} / \mathrm{L})\end{array}$ & $\begin{array}{c}\text { Valor } \\
\text { Calculado }\end{array}$ & $\begin{array}{c}\text { Critério de } \\
\text { aceitabilidade }\end{array}$ \\
\hline \multirow{2}{*}{ Repetitividade } & Hor Rat & 14 & 0,6 & \\
& & 20 & 0,9 & \\
& & 26 & 0,1 & $\leq 2,0$ \\
Recuperação & 32 & 0,9 & \\
& & 2 & 0,7 & \\
& & 8 & 97,5 & \\
& & 14 & 98,9 & \\
& & 20 & 101,8 & \\
& & 26 & 106,5 & \\
& & 32 & 99,0 & \\
& & & 101,4 & \\
\hline
\end{tabular}

Hor Rat $=$ razão de Horwits; $\bar{R}=$ recuperação média

foram realizados (Tabela 3 ).

Conforme apresentado na Tabela 3 , os resultados dos testes permitem afirmar que o método não apresenta efeito matriz, pois, estatisticamente $(\alpha=0,05)$, as curvas analíticas da CAP em solvente e em matriz se equivalem.

\section{Recuperação}

Os valores obtidos (Tabela 4) para a recuperação média em cada nível de concentração encontram-se dentro de limites estabelecidos pela União Européia. ${ }^{9}$

\section{Repetitividade}

Baseando-se em critérios estabelecidos a partir da equação de Horwitz e Albert, os valores de Hor Rat encontrados (Tabela 4) indicam que o método apresenta repetitividade adequada. ${ }^{23}$

\section{Precisão intermediária}

Segundo Horwitz e Albert e União Européia, ${ }^{9,23}$ o valor de $C V(\%)$ igual a 4,6 indica que o método apresenta precisão adequada quando as medidas de uma única amostra são obtidas em dias diferentes.

\section{Limites de detecção e quantificação}

A curva analítica de equação $y=6651,9+17521 x$ foi utilizada para a determinação dos limites. Os limites de detecção e quantificação do método apresentaram valores iguais a 0,83 e $1,63 \mathrm{mg} / \mathrm{L}$, respectivamente. O Codex estabeleceu recentemente critérios de aceitação para LOD e LOQ de um método analítico e estes devem, no máximo, corresponder a 1/10 e 1/5 do limite permitido para uma substância. Dessa forma, os resultados obtidos foram considerados adequados, pois os critérios de aceitação para os limites de detecção e quantificação determinam valores máximos de 1,5 e $3,0 \mathrm{mg} / \mathrm{L} .{ }^{24}$

\section{Adequação do método}

Com base em critérios de aceitabilidade discutidos e ratificados pelo Codex Alimentarius, o método proposto apresentou resultados satisfatórios para todos os parâmetros de desempenho relacionados à validação analítica.

No que tange à aplicabilidade do método ao limite de migração específica da CAP, $15 \mathrm{mg} / \mathrm{L}$, pode-se afirmar que a faixa de trabalho
(2 a $32 \mathrm{mg} / \mathrm{L}$ ) se encontra dentro dos critérios adotados pelo Codex Alimentarius. ${ }^{24}$ Segundo tal referência, as concentrações mínima e máxima do método devem corresponder, respectivamente, ao limite máximo permitido subtraído ou acrescido de três vezes o desvio padrão de reprodutibilidade..$^{24} \mathrm{O}$ desvio padrão de reprodutibilidade é calculado por:

$$
S_{R}=\frac{c \times 2 C^{-0.1505}}{100}
$$

onde: $\mathrm{c}=$ concentração máxima do analito permitida por legislação ou limite máximo permitido (no caso, $15 \mathrm{mg} / \mathrm{L}$ ); C = concentração máxima do analito permitida por legislação expressa em fração (no caso, $15 \times 10^{-6}$ ). Dessa forma, as concentrações mínima e máxima determinadas para o método foram 10,2 e 19,8 mg/L, respectivamente, devendo constar na faixa de trabalho. ${ }^{24}$ Essa condição é plenamente satisfeita, pois o trabalho abrangeu concentrações de 2 a $32 \mathrm{mg} / \mathrm{L}$. Cabe salientar que apesar do critério de aceitabilidade mencionado ser dirigido para validações interlaboratoriais, há o entendimento que o mesmo deve ser observado em validações intralaboratoriais, etapa precursora e fundamental à consecução de estudos colaborativos.

\section{CONCLUSÃO}

O método desenvolvido e validado para a detecção e quantificação da migração de CAP a partir de filmes contendo nylon $6 \mathrm{em}$ simulante etanol $95 \%$ foi considerado adequado ao propósito. Todos os parâmetros avaliados, como linearidade, efeito matriz, repetitividade, precisão intermediária, recuperação e limites de detecção e quantificação, apresentaram resultados satisfatórios. A linearidade foi comprovada na faixa de concentração de 2 a $32 \mathrm{mg} / \mathrm{L}$. Os limites de detecção e quantificação estabelecidos foram de 0,83 e 1,63 mg/L, respectivamente. A repetitividade apresentou valores para Hor Rat inferiores a 2 e a recuperação oscilou entre 97,5 e 106,5\%.

\section{AGRADECIMENTOS}

Ao Instituto Nacional de Controle de Qualidade em Saúde pela disponibilidade de sua estrutura e de seus profissionais reconhecidamente qualificados e à Fundação Oswaldo Cruz pelo apoio financeiro.

\section{REFERÊNCIAS}

1. Abrantes, S. M. P.; Tese de Doutorado, Universidade Federal do Rio de Janeiro, Brasil, 1998.

2. Arvanitoyannis, I. S.; Bosnea, L.; Crit. Rev. Food Sci. Nut. 2004, 44, 63.

3. Félix, J. S.; Monteiro, J.; Manzoli, J. E; Padula, M.; Pezo, D.; Romero, J.; Nerín, C.; Anal. Bional. Chem. 2008, 391, 3.

4. Pogorzelska, Z.; Mielniczuk, Z.; Packag. Technol. Sci. 2001, 14, 1.

5. Bomfim, M. V. J.; Abrantes, S. M. P.; Zamith, H. P. S.; Rev. Bras. Ciênc. Farm. 2009, 45, 1.

6. Brasil. Resolução n ${ }^{\circ} 105$ de 19 de maio de 1999; Diário Oficial da República Federativa do Brasil, Brasília, DF, 20 de maio de 1999.

7. Thompson, M.; Ellison, S. L. R.; Wood, R.; Pure Appl. Chem. 2002, 74, 835.

8. Molecular Simulations Inc.; Web Lab Viewer Pro versão 3.1, San Diego, 1998.

9. União Européia (UE); Commission Directive 2002/72/EC, 06 de agosto de 2002, Official J. European Union 2002, L. 220/18.

10. Comunidade Européia (CE); Council Directive 82/711/CEE, 18 de outubro de 1982, Official J. European Communities 1982, L. 297/26.

11. http://www.anvisa.gov.br/divulga/consulta/index.htm, acessada em Setembro 2009.

12. Souza, S. V. C.; Tese de Doutorado, Universidade Federal de Minas 
Gerais, Brasil, 2007.

13. Instituto Nacional de Metrologia, Normalização e Qualidade Industrial (INMETRO); Orientações sobre Validação de Métodos de Ensaio Químicos, DOQ-CGCRE-008, 2007.

14. International Conference on Harmonisation (ICH); Validation of analytical procedures, Geneva, 1996.

15. Frehse, H.; Thier, H. P.; DFG-Konzept. 1991, 35, 285.

16. Belsey, D. A.; Kuh, E.; Welsch, R. E.; Regression diagnostics: identifying influential data and sources of colinearity, Wiley: New York, 1980.

17. Horwitz, W.; Pure Appl. Chem. 1995, 67, 331.

18. Ryan, T. A.; Joiner, B. L.; Normal probability plots and tests for normality, Pennsylvania State University: Pennsylvania, 1976.

19. Levene, H. Em Contributions to probability and statistics; Olkin, I.; Ghurye, S. G.; Hoeffding, W.; Madow, W. G.; Mann, H. B., eds.; Stanford University Press: California, 1960, cap. 25.

20. Brown, M. B.; Forsythe, A. B.; J. Am. Stat. Assoc. 1974, 69, 364.

21. Durbin, J.; Watson, G. S.; Biometrika 1951, 38, 159.

22. Draper, N. R.; Smith, H.; Applied regression analysis, Wiley: New York, 1998.

23. Horwitz, W.; Albert, R.; J. AOAC Int. 2006, 89, 1095.

24. Procedural Manual, $19^{\text {th }}$ ed, WHO and FAO: Rome, 2010; Report of the thirtieth session of the Codex Committee on methods of analysis and sampling, Hungary, 2009. 